\title{
On the Vertex Multiplication Graphs
}

\author{
M. Saravanan, K. M. Kathiresan
}

Abstract: For any graph $G$, with vertex set $\left\{v_{1}, v_{2}, \ldots, v_{p}\right\}$ and a p-tuble of positive integers $\left(n_{1}, n_{2}, \ldots, n_{p}\right)$, the vertex multiplication graph $G_{n_{1}, n_{2}, \ldots, n_{p}}$ is defined as the graph with vertex set consists of $n_{i}$ copies of each $v_{i} \in V(G)$, where the copies of $v_{i}$ and $v_{j}$ are adjacent in $G_{n_{1}, n_{2}, \ldots, n_{p}}$ if and only if the corresponding vertices $v_{i}$ and $v_{j}$ are adjacent in $G$. In this paper, we prove that the spectrum of $G_{n_{1}, n_{2}, \ldots, n_{p}}$ is same as that of spectrum of its quotient graph $\widetilde{G}_{n_{1}, n_{2}, \ldots, n_{p}}$ with additional zero eigenvalues with multiplicity $n-p$, where $n=n_{1}+$ $n_{2}+\ldots+n_{p}$. Also we prove that the determinant of $\widetilde{\boldsymbol{G}}_{\left\{n_{1}, n_{2}, \ldots, n_{p}\right\}}$ is minimum for $\widetilde{G}_{n-p+1,1, \ldots, 1}$ and maximum for $\widetilde{\boldsymbol{G}}_{\left[\frac{n}{n},,\left|\frac{n}{p}, \ldots, \ldots,\right| \frac{n}{p},\left|, \frac{n}{n},\right|, \frac{n}{p}, \ldots, \mid \frac{n}{p}\right]} \cdot$ Also we find distance- $\boldsymbol{i}$ spectrum of thorn graphs, $G^{+k}$, when diameter 2 .

Keywords : vertex multiplication; eigen values; quotient graph.

\section{INTRODUCTION}

W e follow the terminologies and notations from [6], [19]. Let $G$ be a simple graph with vertex set $\left\{v_{1}, v_{2}, \ldots v_{p}\right\}$ and $\left(n_{1}, n_{2}, \ldots, n_{p}\right)$ be a $p$ - tuble of positive integers. Then the vertex multiplication graph $G_{n_{1}, n_{2}, \ldots, n_{p}}$ is defined as the graph with vertex set consists of $n_{i}$ copies of each $v_{i} \in V(G)$, where the copies of $v_{i}$ and $v_{j}$ are adjacent in $G_{n_{1}, n_{2}, \ldots, n_{p}}$ if and only if the corresponding vertices $v_{i}$ and $v_{j}$ are adjacent in $G$. More precisely, $G_{n_{1}, n_{2}, \ldots, n_{p}}$ is the graph with vertex set $V^{*}$ and edge set $E^{*}$ such that $V^{*}=\cup_{i=1}^{p} V_{i}$ where $V_{i}=\left\{v_{i}^{k} \mid 1 \leq\right.$ $\left.k \leq n_{i}\right\}$ and $E^{*}=\cup_{v_{i} v_{j \in E(G)}}\left\{v_{i}^{l} v_{j}^{m} \mid 1 \leq l \leq n_{i}, 1 \leq m \leq\right.$ $\left.n_{j}\right\}$. Vertex multiplication graphs are studied by various authors in different contexts [14], [16], [19].

Let $G$ be the path on 3 vertices, $P_{3}$, with vertex set $\left\{v_{1}, v_{2}, v_{3}\right\}$ as in Fig.1.

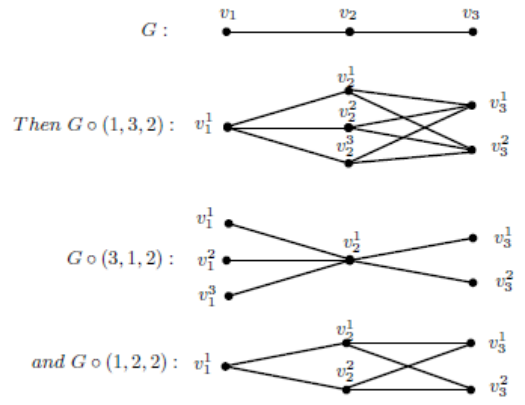

Fig.1. Graph $G$ and its vertex multiplication

Revised Manuscript Received on December 15, 2019

* Correspondence Author

M. Saravanan*, Department of mathematics, Kalasalingam Academy of Research and Education, Krishnankoil,India. Email: msmath.6@gmail.com

K. M. Kathiresan, Director, Centre for Graph Theory, Ayya Nadar Janaki Ammal College, Sivakasi, India. Email: kathir2esan@yahoo.com

If $A(G)$ is the adjacency matrix of $G$ and $\lambda_{1}, \lambda_{2}, \cdots, \lambda_{p}$ are the eigen values of $A(G)$, then the set $\left\{\lambda_{1}, \lambda_{2}, \cdots, \lambda_{p}\right\}$ is called as the spectrum of $G$, denoted by $\operatorname{Spec}(G)$. Suppose $\theta_{1}, \theta_{2}, \cdots, \theta_{k}$ are the distinct eigen values of $G$ and $m_{i}$ is the multiplicity of $\theta_{i}(i=1,2, \ldots, k)$, then thespectrum can be written as the multiset $\left\{\left[\theta_{1}\right]^{m_{1}},\left[\theta_{2}\right]^{m_{2}}, \cdots,\left[\theta_{k}\right]^{m_{k}}\right\}$. The energy $(G)$, of a graph $G$ is defined as the sum of the absolute values of its eigen values, $\varepsilon(G)=\Sigma_{i=1}^{p}\left|\lambda_{i}\right|$. For more on spectrum and energy of graphs, we refer [3], [6], [10], [15].

In [18], the authors proved the following result.

Theorem:Consider complete multipartite graph $K_{n_{1}, n_{2}, \ldots, n_{p}}$ on $n=\sum_{i=1}^{p} n_{i}$ vertices and $n_{1} \geq n_{2} \geq \ldots \geq n_{p}>0$. For fixed value of $n$, spectral radius and energy of $K_{n_{1}, n_{2}, \ldots, n_{p}}$ are minimum for complete split graph $K_{n-p+1,1, \ldots, 1}$ denoted by $C S(n, p-1)$ and maximum for Turan graph $K_{\left.\left[\frac{n}{p}, I, \frac{n}{p}, \ldots, \ldots, \frac{n}{p},, 1 \frac{n}{p}, 1, \frac{n}{p}\right], \ldots, \ldots, \frac{n}{p}\right]}$ denoted by $T(n, p)$.

Now the complete multipartite graph $K_{n_{1}, n_{2}, \ldots, n_{p}}$ can be seen as $K_{p \circ\left(n_{1}, n_{2}, \ldots, n_{p}\right)}$, vertex multiplication of complete graph $K_{p}$ with the tuple $\left(n_{1}, n_{2}, \ldots, n_{p}\right)$ and the result can be modified as spectral radius and energy are minimum for the tuple $(n-p+1,1, \ldots, 1)$ and maximum for the tuple $\left(\left[\frac{n}{p}\right],\left[\frac{n}{p}\right], \ldots,\left[\frac{n}{p}\right],\left[\frac{n}{p}\right],\left\lfloor\frac{n}{p}\right], \ldots,\left\lfloor\frac{n}{p}\right]\right)$.

Motivated by the above result, we are interested in the study of spectrum and energy of $G \circ h$ for general graph $G$ and finding the $p-$ tuples $h$ for which energy and/or spectral radius are/is maximum or minimum. But, as the following examples show, unlike complete multipartite graph, the spectrum may vary for a general vertex multiplication graph $G \circ h$, if the coordinates of $\mathrm{h}$ are rearranged.

Example 1.3. Consider the graph $G=P_{3}$ depicted in Fig.1. Spectrum of its (non isomorphic) vertex multiplication graphs on 6 vertices are given below.

\begin{tabular}{|c|c|c|}
\hline Graph & Edges & Spectrum \\
\hline$G \circ(1,3,2)$ & 9 & $\left\{[3]^{1},[0]^{4},[-3]^{1}\right\}$ \\
\hline$G \circ(2,2,2)$ & 8 & $\left\{[2 \sqrt{2}]^{1},[0]^{4},[-2 \sqrt{2}]^{1}\right\}$ \\
\hline$G \circ(1,2,3)$ & 8 & $\left\{[2 \sqrt{2}]^{1},[0]^{4},[-2 \sqrt{2}]^{1}\right\}$ \\
\hline$G \circ(1,4,1)$ & 8 & $\left\{[2 \sqrt{2}]^{1},[0]^{4},[-2 \sqrt{2}]^{1}\right\}$ \\
\hline$G \circ(4,1,1)$ & 5 & $\left\{[\sqrt{5}]^{1},[0]^{4},[-\sqrt{5}]^{1}\right\}$ \\
\hline
\end{tabular}

spectral radius and energy are maximum for $G^{\circ}(1,3,2)$ and minimum for $G \circ(4,1,1)$.

Example 1.4.For the same graph $G$ in the previous example, spectrum of its (non isomorphic) vertex multiplication graphs on 5 vertices are given below. 


\begin{tabular}{|c|c|c|}
\hline Graph & Edges & Spectrum \\
\hline$G \circ(1,3,1)$ & 6 & $\left\{[\sqrt{6}]^{1},[0]^{3},[-\sqrt{6}]^{1}\right\}$ \\
\hline$G \circ(1,2,2)$ & 6 & $\left\{[\sqrt{6}]^{1},[0]^{3},[-\sqrt{6}]^{1}\right\}$ \\
\hline$G \circ(3,1,1)$ & 4 & $\left\{[2]^{1},[0]^{3},[-2]^{1}\right\}$ \\
\hline
\end{tabular}

spectral radius and energy are maximum for $G^{\circ}(1,3,2)$ and minimum for $G \circ(4,1,1)$.

\section{Example 1.5.}

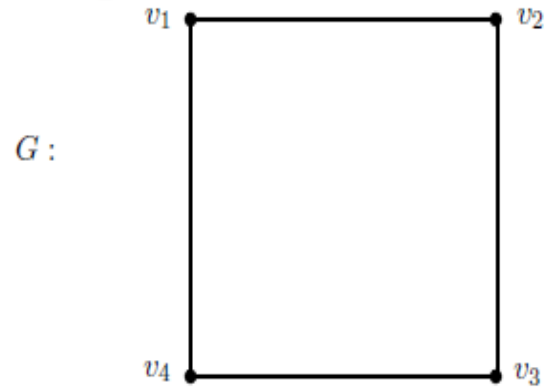

Fig.2: $\mathrm{G}=C_{4}$ with vertex set $\left\{v_{1}, v_{2}, v_{3}, v_{4}\right\}$

Let $G=C_{4}$ be as in Fig.2. spectrum of its (non isomorphic) vertex multiplication graphs on 6 vertices are given below.

\begin{tabular}{|l|l|l|}
\hline Graph & Edges & Spectrum \\
\hline$G \circ(2,2,1,1)$ & 9 & $\left\{[3]^{1},[0]^{4},[-3]^{1}\right\}$ \\
\hline$G \circ(2,1,2,1)$ & 8 & $\left\{[2 \sqrt{2}]^{1},[0]^{4},[-2 \sqrt{2}]^{1}\right\}$ \\
\hline$G \circ(3,1,1,1)$ & 8 & $\left\{[2 \sqrt{2}]^{1},[0]^{4},[-2 \sqrt{2}]^{1}\right\}$ \\
\hline
\end{tabular}

spectral radius and energy are maximum for $G \circ(2,2,1,1)$. $G$ $\circ(3,1,1,1)$ and $G \circ(2,1,2,1)$ are cospectral and spectral radius and energy is minimum for them.

Example 1.6. Consider $G=C_{5}$-vertex multiplication graphs with 8 vertices. spectral radius is maximum for the graph $G \circ(3,2,1,1,1)$ energy is maximum for the graph $G \circ(2,2$, $2,1,1)$. spectral radius and energy are minimum for the graph $G \circ(3,1,2,1,1)$.

Anyway, in the next section we establish relations between spectrum of vertex multiplication graph and its quotient graph.

\section{SPECTRUM OF VERTEX MULTIPLICATION GRAPHS}

Wesaythatapartition $\pi$ of $V(G)$ withcells $C_{1}, C_{2}, \ldots, C_{p}$ isequita bleifthenumberof neighbours in $C_{j}$ ofavertex $u$ of $C_{i}$ isaconstant $b_{i j}$, independentof $u$.Equival entlysubgraphof $G$ inducedbyeachcellisregularandtheedges joininganytwodistinctcellsformasemiregularbipartitegrap h.Now, The directed graph with the $p$ cells of $\pi$ asthe verticesand $b_{i j}$ arcsfrom the $i^{\text {th }}$ to the $j^{\text {th }}$ cells of $\pi$ iscalledquotientor quotient graph of $G$ over $\pi$ denoted by $G / \pi$. Thereforetheentriesoftheadjacencymatrixofthisquo tientaregivenby $A(G / \pi)_{i j}=b_{i j}$. Quotientgraphshavebeenstudi edin[7].

Let $G$ beanygraphon $p$ vertices, withadjacencymatrix $\mathrm{A}=$ $\left[\mathrm{a}_{\mathrm{ij}}\right]$. Let $G_{\mathrm{n} 1, n_{2}, \ldots, n_{p}}$ be the vertex multiplication $\operatorname{graph} G^{\circ}\left(n_{1}\right.$, $\left.n_{2}, \ldots, n_{p}\right)$. Now the vertex partition $\pi=\left\{V_{1}, V_{2}, \ldots V_{p}\right\}$ of $G_{\mathrm{n}_{1}, n_{2}, \ldots, n_{p}}$ is equitable, with $b_{i j}=n_{j} a_{i j}$ for all $\mathrm{i}$ and $\mathrm{j}$.The quotient graph of $G_{\mathrm{n} 1}, n_{2}, \ldots, n_{p} / \pi$ can be denoted as $\widetilde{G}_{\mathrm{n}_{1}, n_{2}, \ldots, n_{p}}$. The adjacency matrix of quotient graph is $\widetilde{G}_{\mathrm{n}_{1}, n_{2}, \ldots, n_{p}}$. is $\mathrm{A}\left(\widetilde{G}_{\mathrm{n}_{1}, n_{2}, \ldots, n_{p} .}\right)=\left[\mathrm{b}_{\mathrm{ij}}\right]$

$$
=\left(\begin{array}{ccccc}
0 & n_{2} a_{12} & n_{3} a_{13} & \ldots & n_{p} a_{1 p} \\
n_{1} a_{21} & 0 & n_{3} a_{23} & n_{p} a_{2 p} \\
& \vdots & & \ddots & \vdots \\
n_{1} a_{p 1} & n_{2} a_{p 2} & n_{3} a_{p 3} & \cdots & 0
\end{array}\right)
$$

To find spectrum of vertex multiplication graph $G \circ \mathrm{h}$, in a particular case $h=(t, t, t, \ldots t)$, we use tensor product of matrices.

Definition 2.1.If $A=\left[a_{i j}\right]$ is a matrix of order $\mathrm{m}_{1} \times \mathrm{n}_{1}$, and $B=\left[b_{i j}\right]$ is a matrix of order $\mathrm{m}_{2} \times \mathrm{n}_{2}$, then the tensor product of $\mathrm{A}$ and $\mathrm{B}$, denoted by $\mathrm{A} \otimes \mathrm{B}$, is a matrix of order $\mathrm{m}_{1} \mathrm{~m}_{2} \times \mathrm{n}_{1} \mathrm{n}_{2}$, obtained by replacing each entry $\mathrm{a}_{\mathrm{ij}}$ of $A$ by the double array $\mathrm{a}_{\mathrm{ij}} \mathrm{B}$.

Theorem 2.2. [6] If $A$ is a matrix of order $m$ with spectrum $\left\{\lambda_{1}, \lambda_{2} \ldots, \lambda_{m}\right\}$, and $\mathrm{B}$ a matrix of order $\mathrm{n}$ with spectrum $\left\{\mu_{1}, \mu_{2}, \ldots, \mu_{n}\right\}$, then the spectrum of $\mathrm{A} \otimes \mathrm{B}$ is $\left\{\lambda_{i} \mu_{j}: 1 \leq i \leq m\right.$; $1 \leq j \leq n\}$.

Theorem 2.3. Let $G$ be a graph on $\mathrm{p}$ vertices with spectrum $\left\{\left[\theta_{1}\right]^{m_{1}},\left[\theta_{2}\right]^{m_{2}}, \ldots,\left[\theta_{k}\right]^{m_{k}}\right\}$.Then the spectrum of $\mathrm{G} \circ \mathrm{h}$ where $\mathrm{h}=(\mathrm{t}, \mathrm{t}, \mathrm{t}, \ldots, \mathrm{t})$ is

$\left\{\left[t \theta_{1}\right]^{m_{1}},\left[t \theta_{2}\right]^{m_{2}}, \ldots,\left[t \theta_{k}\right]^{m_{k}},[0]^{p(t-1)}\right\}$.

Proof. Since $h=(t, t, t, \ldots, t), \mathrm{A}(G \circ \mathrm{h})=\mathrm{A}(G) \otimes \mathrm{J}_{\mathrm{t} \times \mathrm{t}}$, where $\mathrm{J}$ is all one matrix. Now the proof follows from Theorem 2.2 and $\operatorname{Spec}\left(\mathrm{J}_{\mathrm{t} \times \mathrm{t}}\right)=\left\{[\mathrm{t}]^{1},[0]^{\mathrm{t}-1}\right\}$.

Corollary 2.4. $\varepsilon(G \circ \mathrm{h})=t \varepsilon(G)$

But it is hard to find the spectrum of $G \circ h$ for general $h=\left(n_{1}, n_{2}, \ldots, n_{p}\right)$. But we can prove that,

Theorem 2.5.Foranygraph $G$, andap-tuple of positive integers $\left(\mathrm{n}_{1}, \mathrm{n}_{2}, \ldots, \mathrm{n}_{\mathrm{p}}\right)$,

$\operatorname{Spec}\left(G_{\mathrm{n}_{1}, n_{2}, \ldots, n_{p}}\right)=\operatorname{Spec}\left(\widetilde{G}_{\mathrm{n}_{1}, n_{2}, \ldots, n_{p}}\right) \cup\left\{[0]^{n-p}\right\}$.

To prove this theorem, we use a generalization of joinoperation of two graphs, $H$-join of a family of graphs,defined by D. M. Cardesoet all in [5], as follows.

Definition 2.6. Considering the family of graph $G_{1}, G_{2} \ldots G_{\mathrm{p}}$, and the graph $H$, the $H-$ join of $G_{1}, G_{2}, \ldots G_{\mathrm{p}}$ is the graph $G=\mathrm{V}_{\mathrm{H}}\left\{\mathrm{G}_{\mathrm{j}}, \mathrm{j} \in \mathrm{V}(\mathrm{H})\right\}$ such that $\mathrm{V}(G)=\bigcup_{\mathrm{r}=1}^{\mathrm{p}} \mathrm{V}\left(\mathrm{G}_{\mathrm{r}}\right)$ and $\mathrm{E}(\mathrm{G})=\left(\bigcup_{\mathrm{r}=1}^{\mathrm{p}} \mathrm{E}\left(\mathrm{G}_{\mathrm{r}}\right)\right) \mathrm{U}\left(\bigcup_{\mathrm{rs} \in \mathrm{E}(\mathrm{H})}\left\{\mathrm{uv}: \mathrm{u} \in \mathrm{V}\left(\mathrm{G}_{\mathrm{r}}\right), \mathrm{v} \in \mathrm{V}\left(\mathrm{G}_{\mathrm{s}}\right)\right\}\right)$ and they established the following theorem about the spectrum of $H$-join graphs.

Theorem 2.7. [5] Consider the family $\mathcal{F}$ of $\mathrm{p}_{\mathrm{j}}$ regular graphs, $\mathrm{G}_{\mathrm{j}}$ of order $\mathrm{n}_{\mathrm{j}}$,for $\mathrm{j} \in\{1,2, \ldots, \mathrm{k}\}$ and a graph $\mathrm{H}$ such that $\mathrm{V}(\mathrm{H})=\{1,2, \ldots, \mathrm{k}\}$. Then $\left.\operatorname{Spec}\left(\bigvee_{H} \mathcal{F}\right\}\right)=\left(\bigcup_{j=1}^{p}\left(\operatorname{spec}\left(G_{j}\right)-p_{j}\right)\right) \cup \operatorname{Spec}(\widetilde{H}), \quad$ where $\widetilde{H}$ $=\left[\mathrm{h}_{\mathrm{ij}}\right]$ is the $\mathrm{k} \times \mathrm{k}$ matrix defined by

$$
\mathrm{h}_{\mathrm{ij}}= \begin{cases}\sqrt{n_{l} n_{q}} & \text { if } i \neq j \text { and } l q \in E(H) ; \\ 0 & \text { if } i \neq j \text { and } l q \notin E(H) .\end{cases}
$$

and if $\mathrm{i}=\mathrm{j}, \mathrm{h}_{\mathrm{ij}}$ is an element chosen form $\operatorname{Spec}\left(\mathrm{G}_{\mathrm{j}}\right)$.

Published By 
Proof of theorem 2.5:

Let $A=\left[a_{i j}\right]$ be the adjacency matrix of $G$. In the view of definition 2.6, the vertex multiplication graph $\mathrm{G}_{\mathrm{n}_{1, n 2, \ldots, n_{p}}}=G$ 。 $\left(n_{1}, n_{2}, \ldots, n_{p}\right)$, can be seen as G- join of $\overline{K_{n_{1}}}, \overline{K_{n_{2}}}, \ldots, \overline{K_{n_{p}}}$. Hence by applying Theorem 2.7, we get $\operatorname{Spec}\left(\mathrm{G}_{\mathrm{n}_{1}, n_{2}, \ldots, n p}\right)=\left(\bigcup_{j=1}^{p}\left(\operatorname{Spec}\left(\overline{K_{n_{J}}}\right)-\{0\}\right)\right) \cup \operatorname{Spec}(\widetilde{H})$,

Where $\widetilde{H}$ is the following matrix:

$$
=\left(\begin{array}{ccccc}
0 & \sqrt{n_{1} n_{2}} a_{12} & \sqrt{n_{1} n_{3}} a_{13} & \ldots & \sqrt{n_{1} n_{p}} a_{1 p} \\
\sqrt{n_{2} n_{1}} a_{21} & 0 & \sqrt{n_{2} n_{3}} a_{23} & & \sqrt{n_{2} n_{p}} a_{2 p} \\
\sqrt{n_{p} n_{1}} a_{p 1} & \sqrt{n_{p} n_{2}} a_{p 2} & \sqrt{n_{p} n_{3}} a_{p 3} & \cdots & \vdots \\
\vdots
\end{array}\right)
$$

$=\mathrm{DAD}$, where $\mathrm{D}=\left(\begin{array}{ccccc}\sqrt{n_{1}} & 0 & 0 & \ldots & 0 \\ 0 & \sqrt{n_{2}} & 0 & & 0 \\ & \vdots & & \ddots & \vdots \\ 0 & 0 & 0 & \cdots & \sqrt{n_{p}}\end{array}\right)$.

Now the proof follows from, equation (1) and DAD is similar to $\mathrm{ADD}$

Corollary 2.8. Rank of a graph, is the rank of the corresponding adjacency matrix. Then for any positive integers $\mathrm{n}_{1}, \mathrm{n}_{2}, \ldots, \mathrm{n}_{\mathrm{p}}, \quad \operatorname{rank} \quad(\mathrm{G})=\operatorname{rank} \quad\left(\mathrm{G}_{\mathrm{n}_{1}, n_{2}, \ldots, n_{p}}\right)=$ rank $\left(\tilde{G}_{\mathrm{n}_{1, n 2, \ldots, n p}}\right)$

Remark 2.9. In general, if $\mathrm{G}$ is a graph with equitable partition, and $\tilde{G}$ is its corresponding quotient graph, and $\phi(G, \lambda)$ and $\phi(\tilde{G}, \lambda)$ are the respective characteristic polynomials then $\phi(\tilde{G}, \lambda)$ divides $\phi(G, \lambda)$. But if $\mathrm{G}$ is any graph on $\mathrm{p}$ vertices and $h=\left(n_{1}, n_{2}, \ldots, n_{p}\right)$ is a p-tuple of positive integers, then

$$
\phi\left(G_{n_{1}, n_{2}, \ldots, n_{p}}, \lambda\right)=\lambda^{n-p} \phi\left(\tilde{G}_{n_{1}, n_{2}, \ldots, n_{p}}, \lambda\right)
$$

where $n=n_{1}+n_{2}+\cdots+n_{p}$

Theorem 2.10. If $G$ is a graph on $p$ vertices with spectrum $\left\{[\lambda]^{1},[0]^{p-2},[-\lambda]^{1}\right\}$,thenfor any positive integers $\mathrm{n}_{1}, \mathrm{n}_{2}, \ldots, \mathrm{n}_{\mathrm{p}}$

$$
\operatorname{Spec}\left(G_{\mathrm{n}_{1, n 2, \ldots, n p}}\right)=\left\{\left[\sqrt{\left|E^{*}\right|}\right]^{1},[0]^{n-2},\left[-\sqrt{\left|E^{*}\right|}\right]^{1}\right\} .
$$

Proof. Now by Theorem 2.5, Spec $\left(G_{\mathrm{n}_{1}, n_{2}, \ldots, n_{p}}\right)$ will be of the form $\left\{[\theta]^{1},[0]^{p-2},[-\theta]^{1}\right\}$,. Then from the sachs theorem, $-\theta^{2}=\left|E^{*}\right|$ and the proof follows.

\section{DETERMINANT OF QUOTIENT GRAPH}

Though we can not get a similar result as in [18] for the spectral parameters of vertex multiplication graphs, we obtain a similar result for determinant of its quotient graphs.

Note 3.1. Suppose two vertices, in a graph, have same neighbors, then their corresponding rows in the adjacency matrix are equal, and consequently the determinant of the graph will be zero. Hence, instead of Goh we study the determinant of quotient graphs of Goh.

Theorem 3.2. Let $G$ be any graph on $p$ verticesand $G_{n_{1}, n_{2}, \ldots, n_{p}}$ be the vertex multiplication graph $G$ 。 $\left(n_{1}, n_{2}, \ldots, n_{p}\right)$. Then the determinant of the quotient $\operatorname{graph} \widetilde{G}_{\mathrm{n}_{1}, n 2, \ldots, n_{p}}$ is $\left(\prod_{i=1}^{p} n_{i}\right)|G|$.

Proof. If $\mathrm{A}(\mathrm{G})=\left[\mathrm{a}_{\mathrm{ij}}\right]$ and $\mathrm{A}\left(\tilde{G}_{\mathrm{n}_{1}, n_{2}, \ldots, n_{p}}\right)=\left[\mathrm{b}_{\mathrm{ij}}\right]$, then $\mathrm{b}_{\mathrm{ij}}=\mathrm{n}_{\mathrm{j}} \mathrm{a}_{\mathrm{ij}}$ for all $\mathrm{i}$ and $\mathrm{j}$. Hence $\left|\mathrm{A}\left(\tilde{G}_{\mathrm{n}_{1, n}, \ldots, n_{p}}\right)\right|$

$$
=\left|\begin{array}{ccccc}
0 & n_{2} a_{12} & n_{3} a_{13} & & n_{p} a_{1 p} \\
n_{1} a_{21} & 0 & n_{3} a_{23} & \cdots & n_{p} a_{2 p} \\
& \vdots & & \ddots & \vdots \\
n_{1} a_{p 1} & n_{2} a_{p 2} & n_{3} a_{p 3} & \cdots & 0
\end{array}\right|
$$

$=\left(\prod_{i=1}^{p} n_{i}\right)|\mathrm{A}(\mathrm{G})|$

Result 3.3.If $\mathrm{x}$ and $\mathrm{y}$ are any two positive integers, with $x$ $y \geq 2$, then $(x-1)(y+1)>x y$.

Theorem 3.4. Consider the positive integers $n_{1} \geq n_{2} \geq \ldots \geq n_{p}>$ 0 , for which $n=n_{1}+n_{2}+\ldots+n_{\mathrm{p}}$. If $\mathrm{n}$ is fixed, then the product $\prod_{i=1}^{p} n_{i}$ is minimum if $n_{1}=n-p+1$ and $n_{\mathrm{i}}=1$ for all $i>1$ and maximum if $\left(n_{1}, n_{2}, \ldots, n_{\mathrm{p}}\right)=(\lceil n / p\rceil,\lceil n /$ $p, \ldots, n / p, n / p, n / p, \ldots, n / p)$

Proof.Suppose, the product is minimum for $\left(n_{1}, n_{2}, \ldots, n_{p}\right)$ and $n_{i} \geq n_{j} \geq 2$, then $\left(n_{i}+1\right)-\left(n_{j}-\right.$ $1) \geq 2$.Hence by the Result 3.3, $\left(n_{i}\right)\left(n_{j}\right)>\left(n_{i}+\right.$ 1) $\left(n_{j}-1\right)$. Hence $n_{1} n_{2} \ldots n_{i} \ldots n_{j} \ldots n_{p}>n_{1} n_{2} \ldots\left(n_{i}+\right.$ 1)... $\left(n_{j}-1\right) \ldots n_{p}$,contradicting the choice of $\left(n_{1}, n_{2}, \ldots, n_{p}\right)$. Hence all integers $n_{1}, n_{2}, \ldots, n_{p}$ are equal to one, except for one, which is equal to $n-p+1$.

Suppose, the product is maximum for $\left(n_{1}, n_{2}, \ldots, n_{p}\right)$ and for some $i, j, n_{i}-n_{j} \geq 2$, then by Result 3.3, $\left(n_{i}-1\right)\left(n_{j}+\right.$ 1) $>\left(n_{i}\right)\left(n_{j}\right)$. Hence $n_{1} n_{2} \ldots\left(n_{i}-1\right) \ldots\left(n_{j}+1\right) \ldots n_{p}>$ $n_{1} n_{2} \ldots n_{i} \ldots n_{j} \ldots n_{p} \quad, \quad$ contradicting the choice of $\left.\left(n_{1}, n_{2}, \ldots, n_{p}\right)\right)$. Hence $\left|n_{i}-n_{j}\right| \leq 1$ for all $i \neq j$ and so each $n_{i}$ is equal to either $\left\lceil\frac{n}{p}\right\rceil \operatorname{or}\left\lceil\frac{n}{p}\right\rceil$.

Theorem 3.5. Let $n_{1}+n_{2}+\ldots+n_{p}$ be equal to a fixed integern and $G$ be any graph on $p$ vertices, where $p \geq 2$. Then thedeterminant of $\tilde{G}_{n_{1}, n_{2}, \ldots, n_{p}}$ is minimum for $\tilde{G}_{n-p+1,1, \ldots, 1}$ and

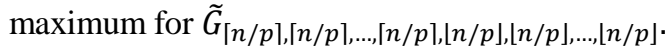

Proof. The proof follows by combining Theorem 3.2 and Theorem 3.4

\section{DISTANCE-iSPECTRUM OF GRAPHS}

The derived graph of a simple graph $\mathrm{G}$, denoted by $G^{+}$,is the graph having the same vertex set as $G$, in which two vertices are adjacent if and only if their distance in $G$ is two . As the notion of derived graph representstheconcept of second electron affinity in inorganicchemistry, the derived graph,its spectrum and itsenergy, called as second stage spectrum and second stage energy respectively, were studied in [2] and continued in [11]. Similarly, the radial graph in[12] and antipodalgraph in [1] of a simple graph G,are the graphs having the same vertex as $\mathrm{G}$,in which two vertices are adjacent if and only if their distance in $\mathrm{G}$ is radius of $\mathrm{G}$ and diameter of $\mathrm{G}$ respectively. A generalization of these concepts,known as distance -i graphs are studied in [4].

Definition4.1.If $G$ is a connected graph, distance- $i$ graph of $G$, denoted by $T_{i}(G)$, is the graph with vertex set as of $G$ and two vertices $x$ and $y$ are adjacent if they are at distance $i$ in $G$

Motivated by second stage spectrum of graphs, we introduce distance $-i$ spectrum of a graph $\mathrm{G}$ as the spectrum of its distance-igraph and denote it by $\operatorname{Spec}_{i}(G)$.For $i=1$,we get usual spectrum, and for $i=2$, we get second stage spectrum. 
Consider a graph $\mathrm{G}$ with vertex $\operatorname{set}\left\{v_{1}, v_{2}, \ldots, v_{p}\right\}$ for every vertex $v_{i}$ attach $\mathrm{k}$ pendant vertices. Then the resulting graph is known as Thorn graph or Thorny graph denoted by $G^{+k}([8],[9]$, and [13]).

In[17], distance $-i$ graphs of cycles, paths and hadamard graphs and their corresponding spectrum have been studied. Here we continue this on study Thorn graphs $G^{+K}$ and find their distance $-i$ spectrum, when $\mathrm{G}$ is connected $\mathrm{r}$-regular graph with diameter 2 .

Proposition 4.2.Let $G$ be graph with vertex set $\left\{\mathrm{v}_{1}, \mathrm{v}_{2}, \ldots, \mathrm{v}_{\mathrm{p}}\right\}$ and $\left(\mathrm{n}_{1}, \mathrm{n}_{2}, \ldots, \mathrm{n}_{\mathrm{p}}\right)$ be a p-tuple of positive integers . For $i \neq 2, T_{i}\left(G_{n_{1}, n_{2}, \ldots, n_{p}}\right)_{i}=T_{i}(G)_{n_{1}, n_{2, \ldots,}} n_{p}$ and for $\mathrm{i}=2, \quad \mathrm{~T}_{2}\left(\mathrm{G}_{\mathrm{n}_{1}, \mathrm{n}_{2, \ldots,}, \mathrm{n}_{\mathrm{p}}}\right)=\mathrm{T}_{2}(\mathrm{G})_{\mathrm{n}_{1}, \mathrm{n}_{2, \ldots, \ldots}} \mathrm{n}_{\mathrm{p}}$ with additional edges, that form cliques on copies of same vertex. That is,replace each vertex $v_{i}$ of $T_{2}(G)$ by $K_{n_{1}}$ and all possible edges.

Proof. Let $u$ and $v$ be its two vertices of $\mathrm{G}$ and $u^{\prime}$ and $v^{\prime}$ be their Copies in $G_{n_{1}, n_{2, \ldots}, n_{p}}$. Then $d\left(u^{\prime}, v^{\prime}\right)=d(u, v)$, if $u \neq v$, and $d\left(u^{\prime}, v^{\prime}\right)=2$, if $u=v$. Hence the proof follows from the definition of distance- $-\mathrm{i}$ graph.

Lemma 4.3. [6] Let $A, B, C$ and $D$ be matrices and let $M=\left[\begin{array}{ll}A & B \\ C & D\end{array}\right]$.Then $|M|=|A D-B C|$, if $A$ and $C$ commutes

Lemma 4.4.[6]Let Gbe a connected r-regular graph on $\mathrm{p}$ vertices with an adjacency matrix $A$ having $m$ distinct eigenvalues $\lambda_{1}=r, \lambda_{2}, \ldots, \lambda_{m}$. Then,thereexistsapolynomial $\mathrm{Q}(\mathrm{x})=\mathrm{p} \frac{\left(\mathrm{x}-\lambda_{2}\right)\left(\mathrm{x}-\lambda_{3}\right) \ldots\left(\mathrm{x}-\lambda_{\mathrm{m}}\right.}{\left(\mathrm{r}-\lambda_{2}\right)\left(\mathrm{r}-\lambda_{3}\right) \ldots\left(\mathrm{r}-\lambda_{\mathrm{m}}\right)}$ such that $\mathrm{Q}(\mathrm{A})=\mathrm{J}$ so that $\mathrm{Q}(\mathrm{r})=\mathrm{p}$ and $\mathrm{Q}\left(\lambda_{\mathrm{i}}\right)=0 \forall \lambda_{\mathrm{i}} \neq \mathrm{r}$

Theorem 4.5: Let $\mathrm{G}$ be a connected $\mathrm{r}$ - regular graph with diameter 2 on $p$ vertices with $\operatorname{spec}(G)=\left\{\lambda_{1} \geq \lambda_{2} \geq \cdots \geq \lambda_{p}\right\}$. Then for $\mathrm{k} \geq 1$, distance-i spectrum of $G^{+k}$ are given as below:

$\operatorname{Spec}_{1}\left(G^{+k}\right)$ consists of

$$
\begin{gathered}
\frac{1}{2}\left[\lambda_{i} \pm \sqrt{\lambda_{i}^{2}+4 k}\right] \text { with multiplicity } 1 \text { for } \mathrm{i}=1 \text { to } \mathrm{p} \\
0 \text { with multiplicity } p(k-1)
\end{gathered}
$$

$\operatorname{Spec}_{2}\left(G^{+k}\right)$ consists of

$$
\begin{gathered}
\frac{1}{2}\left[k-1+p \pm \sqrt{(k-1+p)^{2}-4\left(p k-p-k r^{2}\right)}\right. \text { with } \\
\text { multiplicity } 1 ; \\
\frac{1}{2}\left[k-1 \pm \sqrt{(k-1)^{2}+4 k \lambda_{i}^{2}} \text { with multiplicity1for } \mathrm{i}=2\right. \text { to } \\
\mathrm{p} ;
\end{gathered}
$$

-1 with multiplicity $p(k-1)$.

$\operatorname{Spec}_{3}\left(G^{+k}\right)$ consists of

$$
\begin{gathered}
\frac{1}{2}\left[k r \pm \sqrt{(k r)^{2}+4 k\left(p-1-r^{2}\right)}\right] \text { with multiplicity } 1 \\
\frac{1}{2}\left[k \lambda_{i} \pm \sqrt{\left(k \lambda_{i}\right)^{2}+4 k\left(1+\lambda_{i}\right)^{2}}\right] \text { with multiplicity1for } \mathrm{i}=2 \\
\text { to } \mathrm{p} ; \\
0 \text { with multiplicity } p(k-1) \\
\operatorname{Spec}_{4}\left(G^{+k}\right) \text { consists of } \\
k(p-1-r) \text { with multiplicity } 1 \\
k\left(-1-\lambda_{i}\right) \text { with multiplicity1for } \mathrm{i}=2 \text { to } \mathrm{p} \\
0 \quad \text { with multiplicity } p k
\end{gathered}
$$

Proof: First we consider the case $\mathrm{k}=1$, that is the graph $G^{+1}$. Let $\left\{v_{1}, v_{2}, \ldots, v_{p}\right\}$ be the vertices of $\mathrm{G}$ and $\left\{v_{p+1}, v_{p+2}, \ldots, v_{2 p}\right\}$ be the corresponding pendent vertices. Let $A$ and $\bar{A}$ be the adjacency matrices of $G$ and $\bar{G}$ respectively. Adjacency matrices of $T_{i}\left(G^{+1}\right)$ are

$\left[\begin{array}{ll}A & I \\ I & 0\end{array}\right],\left[\begin{array}{cc}\bar{A} & A \\ A & 0\end{array}\right],\left[\begin{array}{cc}0 & \bar{A} \\ \bar{A} & A\end{array}\right]$ and $\left[\begin{array}{ll}0 & 0 \\ 0 & \bar{A}\end{array}\right]$ for $\mathrm{i}=1,2,3,4$ respectively. Now $G^{+k}=G_{n 1, n 2, \ldots n 2 p}^{+1}$ where $n_{j}=1$ and $n_{p+j}=k$ for $j=1,2, \ldots, p$. Hence from Theorems 2.5,2.7 and Proposition 4.2, spectrum of $T_{i}\left(G^{+k}\right)$ are $\left\{[0]^{p(k-1)}\right\} \cup$ $\operatorname{spec}\left[\begin{array}{cc}A & k I \\ I & 0\end{array}\right], \quad\left\{[-1]^{p(k-1)}\right\} \cup \operatorname{spec}\left[\begin{array}{cc}\bar{A} & \sqrt{k A} \\ \sqrt{k A} & (k-1) I\end{array}\right]$, $\left\{[0]^{p(k-1)}\right\} \cup \operatorname{spec}\left[\begin{array}{ll}0 & k \bar{A} \\ \bar{A} & k A\end{array}\right] \quad$ and $\quad\left\{[0]^{p(k-1)}\right\} \cup$ $\operatorname{spec}\left[\begin{array}{cc}0 & 0 \\ 0 & k \bar{A}\end{array}\right]$ for $\mathrm{i}=1,2,3,4$ respectively. Now the proof follows from Lemmas 4.3 and 4.4.

\section{ACKNOWLEDGMENT}

The first author thanks National Board for Higher Mathematics, DAE,India for supporting him by NBHM Ph.D. fellowship (Grant number2/39(2)/2009/NBHM/R\& D-II/1941), since a part of this work is done by him during his Ph.D.

\section{REFERENCES}

1. R. Aravamuthan, B. Rajendran, On antipodal graphs, Discrete Mathematics, 49(1984) 193-195.

2. S. K. Ayyaswamy, S. Balachandran, I. Gutman, On Second stage spectrum and Energy of a graph, Kragujevac Journal of Mathematics, 34(2010) 139-146.

3. R. Balakrishnan, The energy of a graph, Linear Algebra and its Applications, 387(2004) 287-295.

4. A. E. Brouwer, A. M. Cohen, A. Neumair, Distance Regular graphs, Springer - verlag, NewYork, 1989.

5. D.M. Cardoso, M.A.A. de Freitas, E.A. Martins, M. Robbiano, Spectra of graphs obtained by a generalization of the join graph operation Discrete Mathematics, 313 (2013) 733-741.

6. D. Cvetkovic, M. Doob, H. Sachs, Spectra of Graphs : Theory and Application, Academic Press, New York, 1980.

7. C. Godsil, G. Royle, Algebraic graph theory, Springer - verlag, NewYork, Inc, 2001.

8. E I. Gutman, Generalizations of recurrence relation for the characteristic polynomial of trees, Publ. Inst. Math. (Beograd) 63 (1998) 31-36.

9. I. Gutman, Distance in thorny graph, Publ. Inst. Math. (Beograd) 21(1977) $75-80$

10. I. Gutman, in The Energy of a Graph: Old and New Results, in: A.Beten A. Kohnert, R. Laue, A. Wassermann (eds.) Algebraic Combinatorics and Applications, Springer - verlag, Berlin 2001,196-211

11. S. R. Jog, S. B. Hande, I. Gutman, S. B. Bozkurt, Derived graphs of some graphs, Kragujevac Journal of Mathematics, 36 (2012) 309-314

12. KM. Kathiresan, G. Marimuthu, A study on radial graphs, Ars Combinatoria, 96 (2010) 353-360.

13. D. J. Klein, T. Doslic and D. Bonchev, Vertex-weightings for distance moments and thorny graphs, Discr. Appl. Math., 155 (2007), 2294-2302.

14. K.M. Koh, E.G. Tay, On optimal orientations of $G$ vertexmultiplications, Discrete Mathematics, 219 (2000) 153-171.

15. X. Li, Y. Shi, I. Gutman, Graph Energy, Springer, New York, 2012.

16. K.L. Ng, K.M. Koh, On optimal orientation of cycle vertex multiplica tions, Discrete Mathematics 297 (2005) 104-118.

17. M. Saravanan, KM. Kathiresam, On the energy of Hadamard Graph, Bull. Inst. Combin. Appl. 76(2016), 16-28.

18. D. Stevanovic, I. Gutman, M.U. Rehman, On spectral radius and energy of complete multipartite graphs, Ars Mathematica Contemporanea 9 (2015) 109-113.

19. D. B. West, Introduction to Graph Theory, Prentice Hall, Upper Saddle River, New Jersey, 2000. 


\section{AUTHORS PROFILE}

M. Saravanan has done his Ph.D. in Spectral Graph theory.

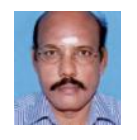

K. M. Kathiresan had done his Ph.D. in Graph theory. $\mathrm{He}$ has published more than 80 articles in reputed journals. He has served as reviewer in many reputed journals and he is the member of many academic societies. Also he has received "TANSA - 2012 Award (Tamilnadu Scientist Award)" awarded by TNSCST. 